\title{
Design and Simulation of the Controller Proportional - Integral - Differential (PID) for Unmanned Surface Vessels
}

\author{
Nhat Duy Nguyen ${ }^{1}$, Thanh Lanh Le ${ }^{2}$, Nam Thoi Le ${ }^{3}$ \\ ${ }^{1,2,3}$ Dong Nai Technology University
}

\begin{tabular}{l}
\hline Article Info \\
\hline Article history: \\
Received Oct 12, 2021 \\
Revised Dec 10, 2021 \\
Accepted Dec 25, 2021 \\
\hline
\end{tabular}

\section{Keywords:}

Guidance

Unmanned Surface Vessels

PID

Controller

\begin{abstract}
Unmanned Surface Vessels (USVs) are devices that use advanced, automated technologies for control and monitoring, with very little human intervention in this system. Today's un drone vehicles are developed for security, defense, trade, and civilian life purposes. Unmanned Surface Vessels is a device that uses advanced and automated technologies to control and monitor. This paper identifies the proportional - integral - differential controller. Results using MATLAB / SIMULINK simulation software.
\end{abstract}

This is an open access article under the CC BY-SA license.

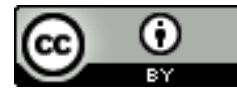

\section{Corresponding Author:}

Nhat Duy Nguyen,

Dong Nai Technology University,

Nguyen Khuyen Street, Ward 5, Trang Dai Ward, City. Bien Hoa, Dong Nai, Vietnam.

Email:nguyennhatduy@dntu.edu.vn

\section{INTRODPUCTION}

Vietnam is a coastal country with a coastline of more than $3000 \mathrm{~km}$, waters three times larger than the mainland with many island and archipelago systems, so economic, scientific, tourism and sovereignty protection activities at sea are very active. A series of works such as bumper bridges, drilling rigs, oil pipelines has been and is being built at sea. In the process of construction and exploitation of the above works, there has been a huge demand for conducting exploration, surveying and conducting works both above and below.

All the above tasks require a device capable of continuous, automatic performance for large workloads. These tasks may be in dangerous environments such as wind waves, extreme weather, polluted environments. Despite this, these tasks are usually human in charge, which in doing so can reduce performance as well as increase costs. One solution that has been proposed is to use unmand boats. The vehicle is self-capable and is easily combined with other specialized equipment to perform a variety of specialized tasks and can also perform many tasks simultaneously. Within the framework of the article, it will mention the construction of PID controllers for uns manless ships on water for security, defense, trade and civilian life. On that basis will conduct research and design an automatic operating marine equipment with the goal of testing, testing the combination of navigation system and automatic control system. 


\section{OVERVIEW OF THE CONTROLLER PROPORTIONAL - INTEGRAL - DIFFERENTIAL (PID)}

The controller proportional - integral - differential (PID) used extensively because of its simple structure. The output of the PID controller depends on the input and the parameters according to the formula (Figure 1):

$$
u(t)=K_{p} e(t)+K_{i} \int e(t) d t+K_{d} \frac{d e(t)}{d t}
$$

in which: $e(t)$ - error, determined by the signal of the desired output and the measured output $e(t)=y_{d}(t)-y(t)$

$K_{p}, K_{i}, K_{d}$ - ratio, analysis, differentially. These systems have a mutual influence and affect the quality of the controller

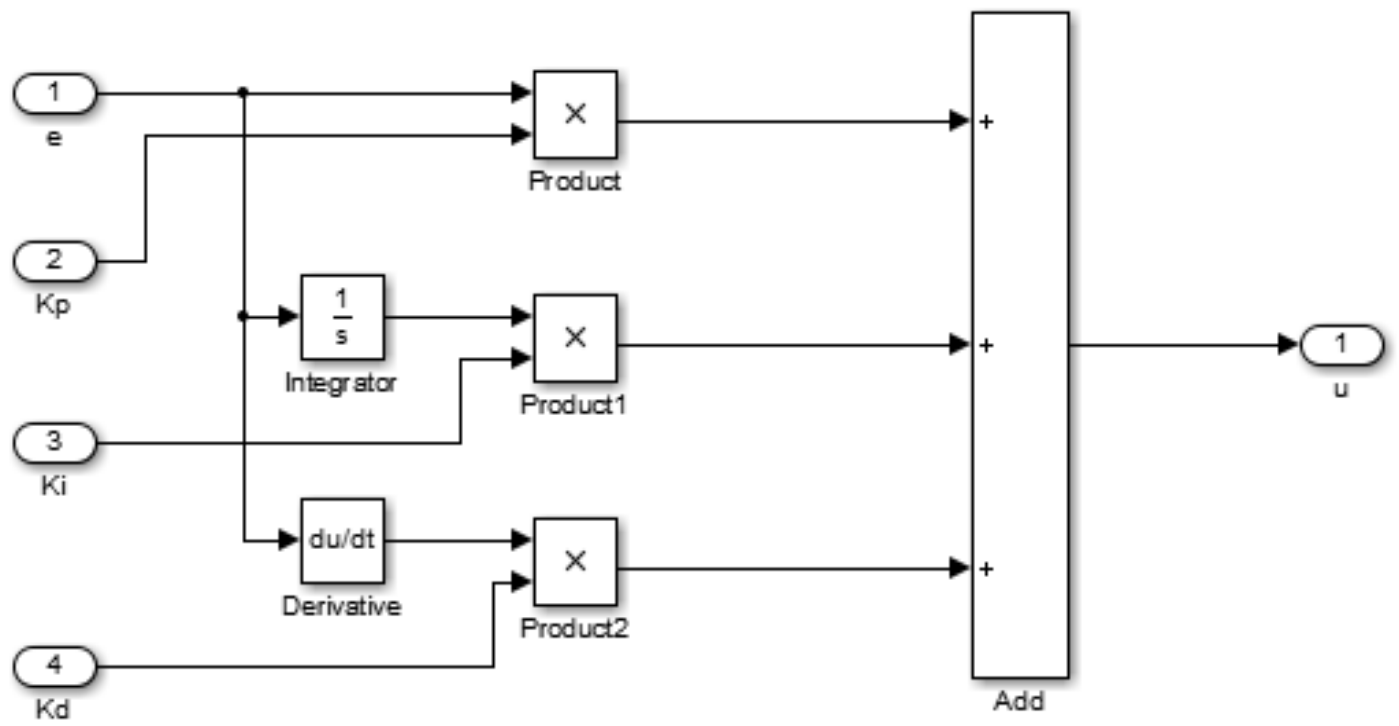

Figure 1. Configure controllers PID in MATLAB/SIMULINK

PID controllers have a simple structure, however this controller only gives good responsive ability to linear models. In fact, models often have nonlinear components, so PID controllers usually only give a good response at certain work points, the remaining quality points are often inferior. At that time, it will be necessary to use the sliding controller (Sliding Mode Control) is a nonlinear controller (this will be mentioned in another article).

\section{VESSELS CONTROL CHARACTERISTICS}

\subsection{Autopilots}

Autopilots (auto steering) means equipment that controls aircraft, ships or other means without human intervention. Autopilots was intended to maintain control, ability to hold the course, and was used to replace the pilot on the ships during the cruise. In addition, modern Autopilots can perform other operations, such as moving or parking, or allowing control of unstable vessels such as submarines and some large tankers.

\subsection{Waypoint Tracking and Path-Following Control Systems}

Within the framework of the article, a controller will be researched and designed to solve the problem

International Journal of Research in Vocational Studies, Vol. 1, No. 4, January 2022: 01 - 10 
of sticking the road with the given Waypoint points. The line drawing will be solved in the Guidance system. Once there is a path and the data from the Guidance system, we will control the train to follow that path.

\subsection{The principles of Guidance, Navigation and Control}

A motion control system is built consisting of three independent blocks in which it is in turn Guidance, Navigation and Control (GNC). These systems interact with each other through data and signal transmission as illustrated in Figure 2. In more advanced GNC systems, these blocks can be more closely connected and even represented by a block.

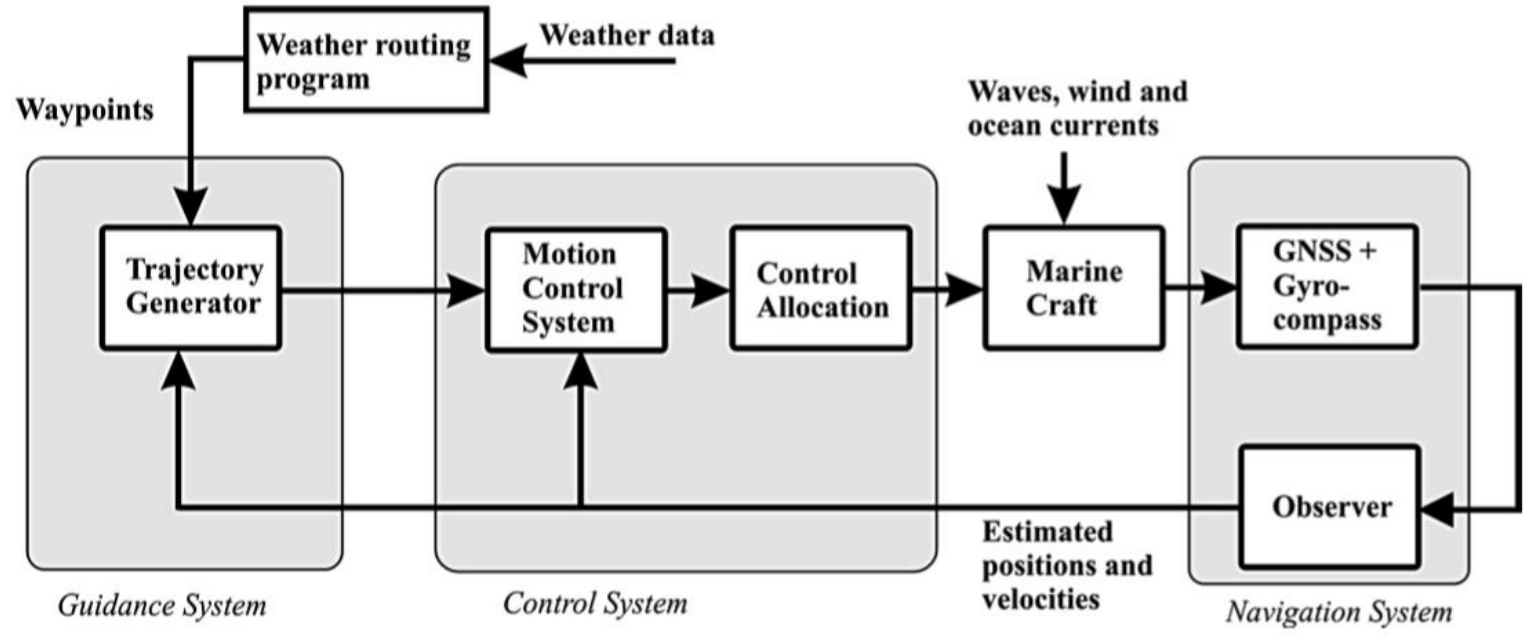

Figure 2. Diagram showing the link of a GNC

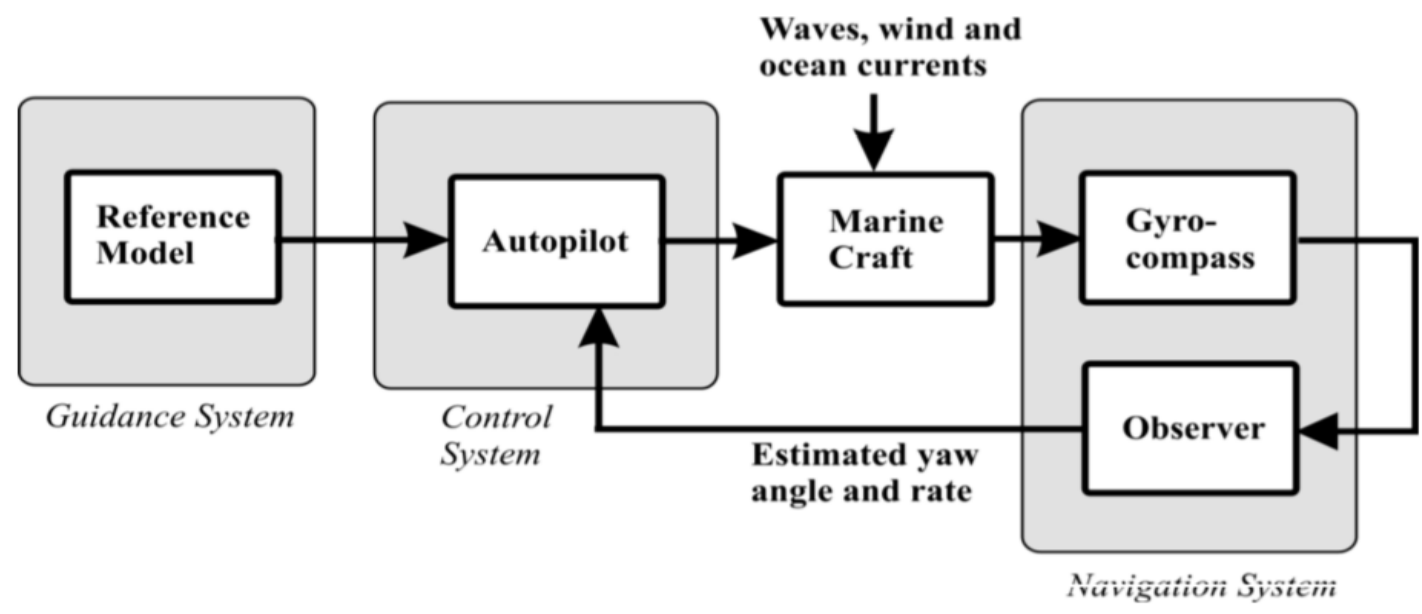

Figure 3. Diagram linking GNC with navigation system is open loop

In Figure 2, the guidance system uses estimated positions and velocity. This is a closed loop guidance system while a navigation system that uses only reference feeds (without feedback) is an open loop guidance system (Figure 3).

In the diagram in Figure 3, the basic blocks have the following functions and tasks:

- Guidance System: the system controls the movement of the vessel, which constantly calculates parameters such as the reference position, speed and acceleration of the vessel. These parameters are usually 
provided to operators and navigation systems. The basic components of the navigation system are motion sensors, external data such as weather data (wind speed and direction, height and slope, speed and wave direction) and computers. The computer collects and process information, and then loads the results into the motion control system.

- Navigation System: is the technique of driving a train by determining its location, path and distance of travel. In some cases, velocity and acceleration are also determined. This is done using a global positioning satellite system in combination with motion sensors such as acceleration and the switch.

- Control System: The control system is responsible for determining the force and control torque required that the vessel must provide to meet a certain control objective. Desired control objectives are often seen together with the guidance system.

\section{DESIGN AND SIMULATION OF CONTROL ALGORITHMS USING PID}

First, we will build a common mathematical model to use different algorithms for the design of controllers as well as favorable in comparing and evaluating algorithms with each other later.

The equation used to simulate the control algorithm using PID is:

$$
\left\{\begin{array}{l}
\dot{\eta}=R(\psi) v \\
M \dot{v}+D(v) v=\tau
\end{array}\right.
$$

In which

$\eta=[x, y, \psi]^{T}$ in the coordinate system NED (NED is the coordinate system often mentioned in everyday life).

$v=[u, v, r]^{T}$ in the coordinate system BODY (BODY is a mode of movement fixed to a ship).

$\tau=\left[\tau_{1}, \tau_{2}, \tau_{3}\right]^{T}=B \alpha$, với $B$ is the control matrix, $\alpha$ is a control signal. Since the vessel is only driven by vertical thrust and rotating torque i.e. $\tau_{1}$ and $\tau_{3}$, but when there is a component $\tau_{3}$ will create $\tau_{2}$, so you can rewrite $\tau=\left[\tau_{1}, A \tau_{3}, \tau_{3}\right]^{T}$ with $A$ is the ratio.

$$
R(\psi)=\left[\begin{array}{ccc}
\cos \psi & -\sin \psi & 0 \\
\sin (\psi) & \cos (\psi) & 0 \\
0 & 0 & 1
\end{array}\right]+\left[\begin{array}{ccc}
m_{11} & 0 & 0 \\
0 & m_{22} & m_{23} \\
0 & m_{32} & m_{33}
\end{array}\right]=\left[\begin{array}{ccc}
m-X_{\dot{u}} & 0 & 0 \\
0 & m-Y_{\dot{u}} & m x_{G}-Y_{\dot{r}} \\
0 & m x_{G}-N_{\dot{v}} & I_{z}-N_{\dot{r}}
\end{array}\right]
$$

with $\left[x_{G}, y_{G}, z_{G}\right]$ is the central coordinate of the vessel in the BODY coordinate system; $I_{z}$ is the inerity matrix in which the torque rotates around the ship's axis; are the numbers in the Added Mass matrix, these systems depend on the shape of the vessel $X_{\dot{u}}, Y_{\dot{v}}, N_{\dot{r}}$. 


$$
\begin{aligned}
& D(v)=D+D_{n}(v)=\left[\begin{array}{ccc}
d_{11} & 0 & 0 \\
0 & d_{22} & d_{23} \\
0 & d_{32} & d_{33}
\end{array}\right] \text {, với } D=\left[\begin{array}{ccc}
-X_{u} & 0 & 0 \\
0 & -Y_{v} & -Y_{r} \\
0 & -N_{v} & -N_{r}
\end{array}\right] \text { and } \\
& D_{n}(v)=\left[\begin{array}{ccc}
-X_{|u| u}|u| & 0 \\
0 & -Y_{|v| v}|v|-Y_{|r| v}|r| & -Y_{|v| r}|v|-Y_{|r| r \mid}|r| \\
0 & -N_{|v| v}|v|-N_{|r| v}|r| & -N_{|v| r \mid}|v|-N_{|r| r \mid}|r|
\end{array}\right]
\end{aligned}
$$

Here, parameters of $D_{n}(v)$ often shortened or ignored during calculations when the train moves at a slow speed. However, we will keep to survey the nonlinearity of the system.

\subsection{PID Controller Design}

The following will divide the control problem into two small problems including:

- Control direction and stick to the desired angle (Autopilots).

- Control the speed in the strait of the train (Speed Assignment).

Therefore, it will take two PID controllers to solve the above two problems. From the dynamic properties we will choose the controller that keeps the direction as PD and keeps the speed as PI.

Deploy (2) we have:

$$
\left\{\begin{array}{l}
\dot{x}=u \cos \psi-v \sin \psi \\
\dot{y}=u \sin \psi+v \cos \psi \\
\dot{\psi}=r \\
\left(m-X_{\dot{u}}\right) \dot{u}-X_{u} u-X_{|u| u}|u| u=\tau_{1} \\
\left(m-Y_{\dot{v}}\right) \dot{v}+\left(m x_{G}-Y_{\dot{r}}\right) \dot{r}-\left(Y_{v}+Y_{|v| v}|v|+Y_{|r| v \mid}|r|\right) v-\left(Y_{r}+Y_{|v| r \mid}|v|+Y_{|r| r}|r|\right) r=A \tau_{3} \\
\left(m x_{G}-N_{\dot{v}}\right) \dot{v}+\left(I_{z}-N_{\dot{r}}\right) \dot{r}-\left(N_{v}+N_{|v| v}|v|+N_{|r| v \mid}|r|\right) v-\left(N_{r}+N_{|v| r}|v|+N_{|r| r \mid}|r|\right) r=\tau_{3}
\end{array}\right.
$$

In the above system of equations, the first three are dynamic equations that express the position of the vessel in the NED system, while the next three are the dynamic equations in the BODY system.

We have a diagram that simulates the PID controller shown on Figure 4. 


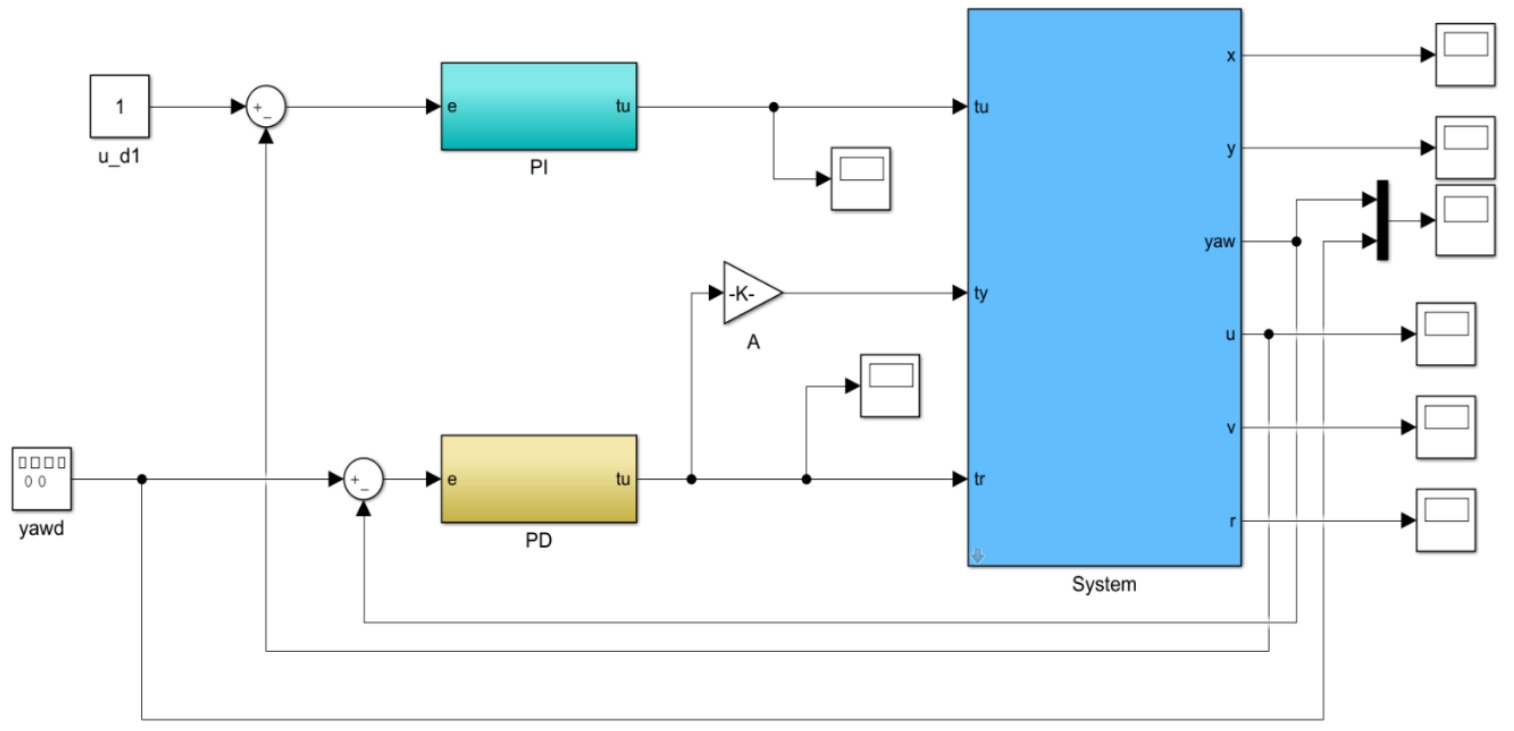

Figure 4. PID controller simulation diagram.

The block diagram of the system is the mathematical model of the vessel shown on Figure 5.

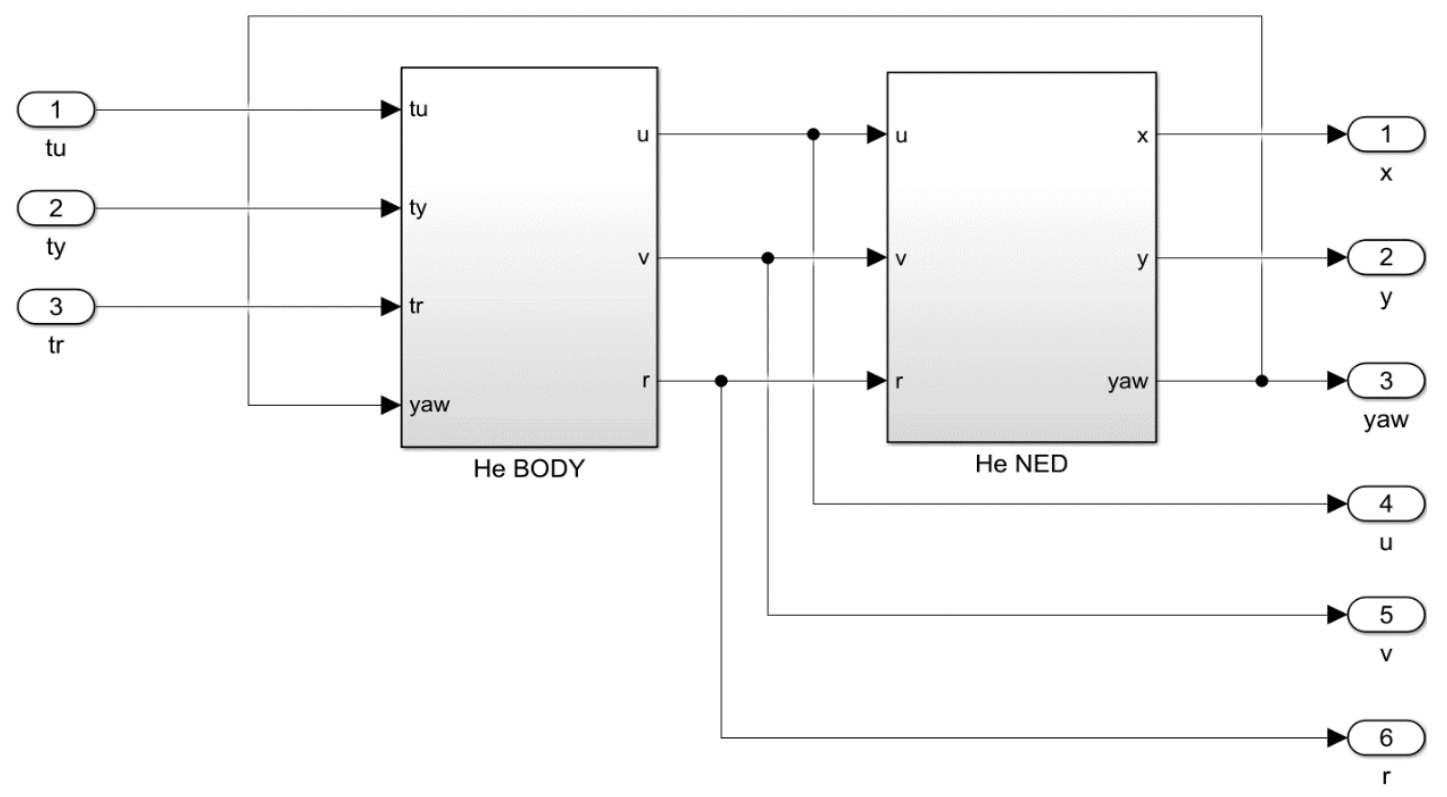

Figure 5. System Block Diagram

\subsection{PID controller simulation results}

The parameters used for simulation are as follows: single-hull vessels have a mass of $15 \mathrm{~kg}$ and a length of $1.255 \mathrm{~m}$. The way the control only involves the thrust impact on $\tau_{1}$ and momen turn $\tau_{3}$.

$$
D=\left(\begin{array}{ccc}
n_{11} & 0 & 0 \\
0 & n_{22} & n_{23} \\
0 & n_{32} & n_{33}
\end{array}\right)=\left(\begin{array}{ccc}
2 & 0 & 0 \\
0 & 7 & 0.1 \\
0 & 0.1 & 0.5
\end{array}\right)
$$




$$
M=\left(\begin{array}{ccc}
m_{11} & 0 & 0 \\
0 & m_{22} & m_{23} \\
0 & m_{32} & m_{33}
\end{array}\right)=\left(\begin{array}{ccc}
25.8 & 0 & 0 \\
0 & 33.8 & 1.0115 \\
0 & 1.0115 & 2.76
\end{array}\right)
$$

Find parameters for two PI and PD controllers, first propose yawd $=0$ (i.e. no force or $\tau_{3}=\tau_{2}=0$ ) and set the desired velocity for the vessel to find parameters for the PI velocity.

Coefficient $K_{p}$ and $K_{i}$ selected 20 and 18, and thrust limits of 20N. The velocity response result is simulated in MATLAB/SIMULINK and shown in Figure 6.

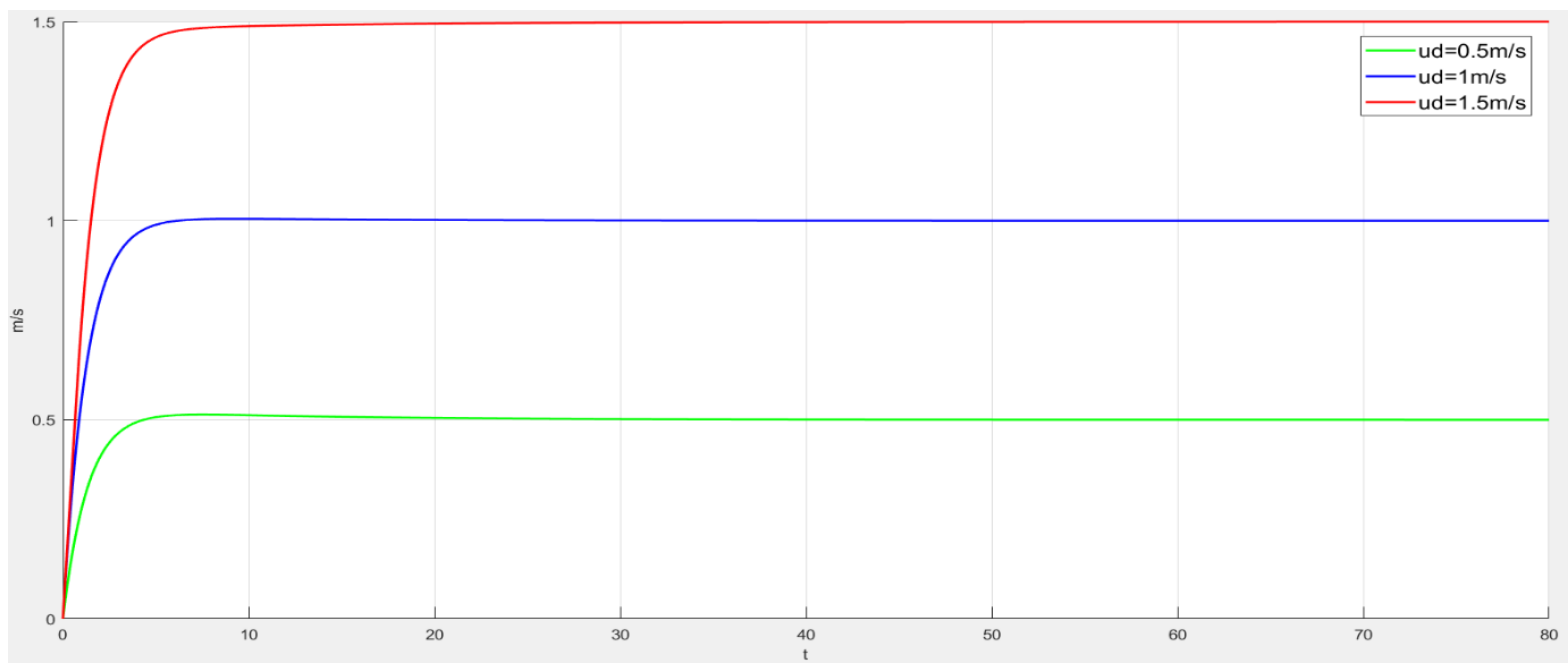

Figure 6. Simulate velocity at different set values of the PI controller

Through Figure 6, there is an effect on the quality of the PI controller (decreases when $u_{d}$ change) due to the high nonlinearity of the system (damping system) and the maximum thrust limit from thruster $(\max =$ $20 N)$.

Similarly, select $u_{d}=0$ and find parameters for the PD set. Coefficient $K_{p}$ and $K_{d}$ selected 20 and 20, for moment limits of $10 \mathrm{Nm}$, I has the simulation results as shown in Figure 7.

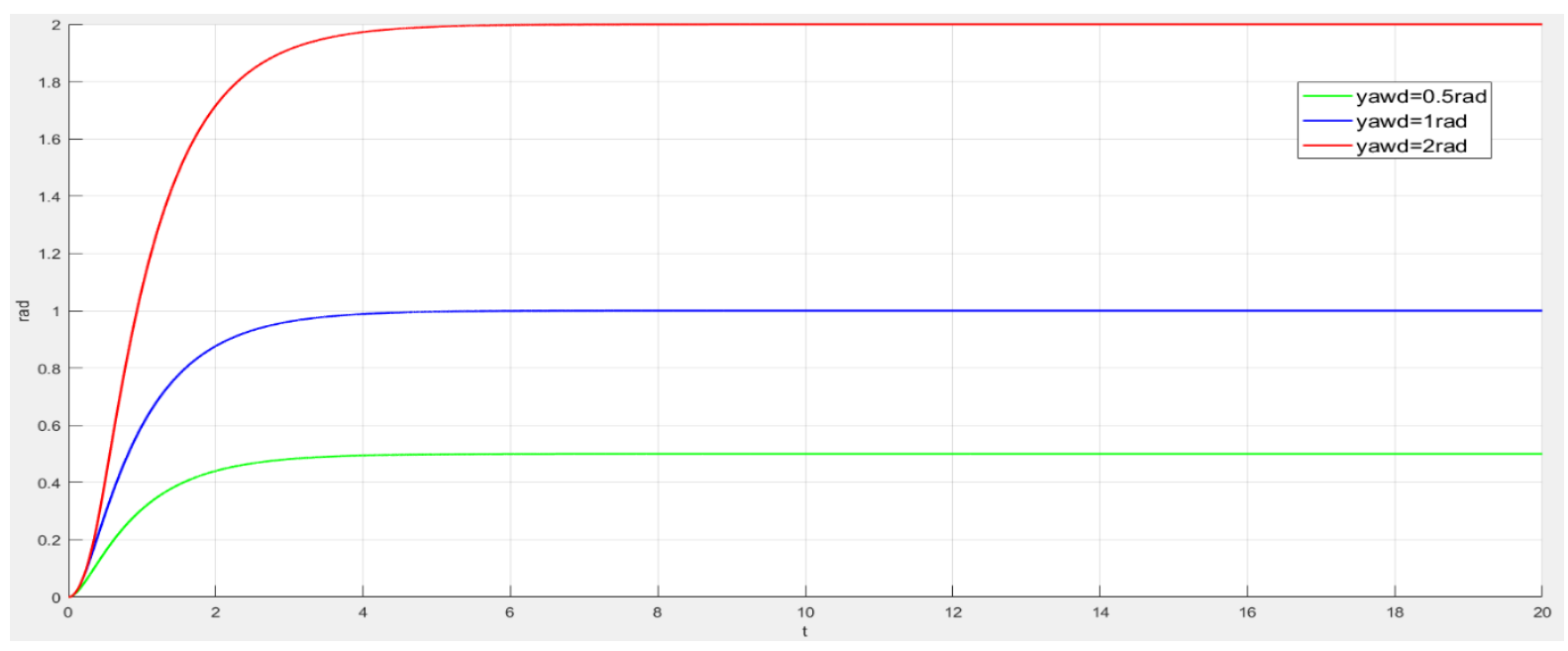

Figure 7. Simulate yaw angles at different set values of PD controllers 
Through figure 7 with comments, the quality of the PD set is hardly affected much when changing yawd. This can be drawn from the Nomoto equation for steering wheel boat control (which can be linearized and written as a transmission function).

The above assessment is independent, so to fully consider we will place both the desired velocity and yaw angle with $u_{d}=1 \mathrm{~m} / \mathrm{s}$ and yawd is a square wave with an amplit margin of 1 (Figure 8 ).

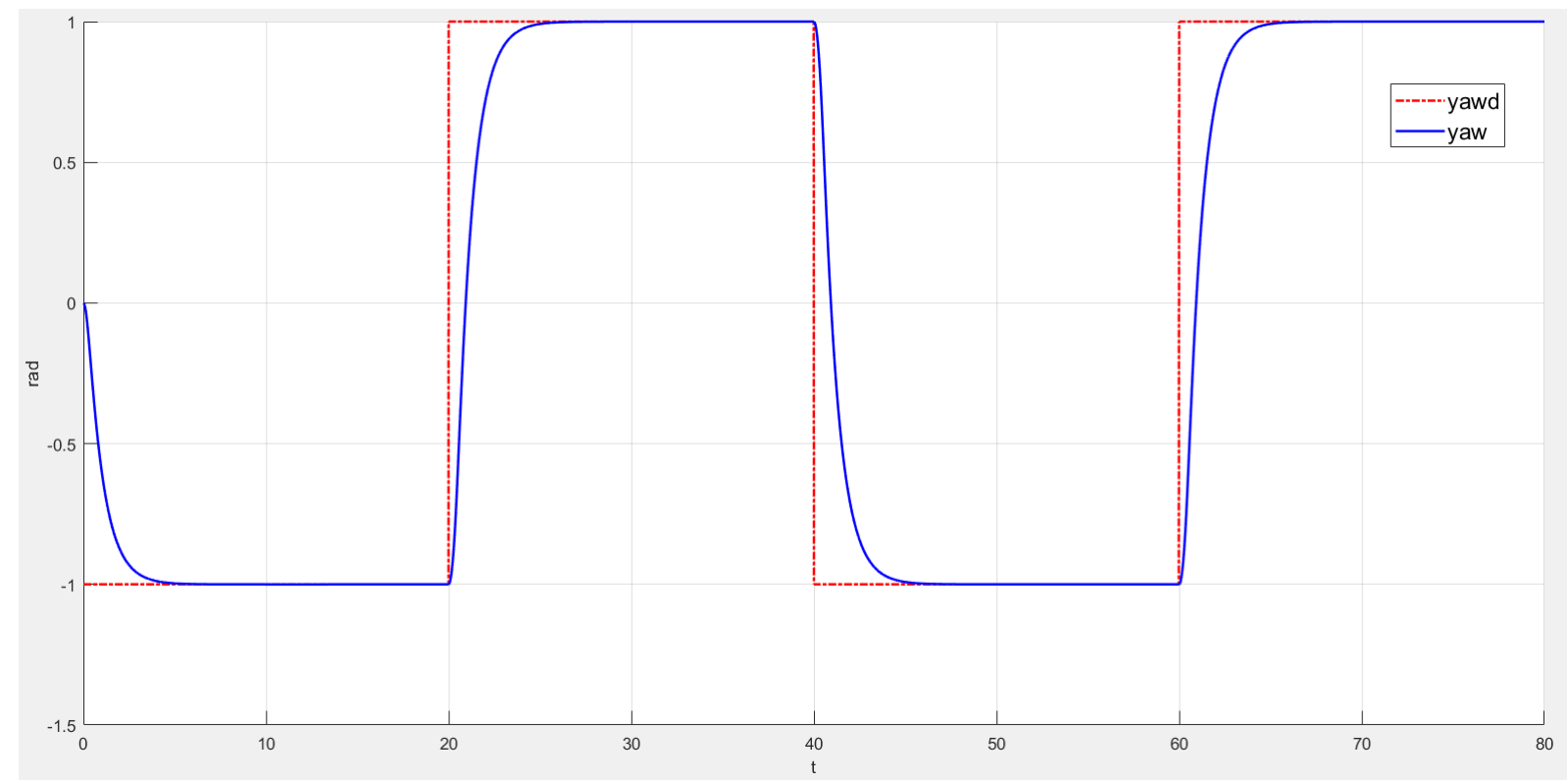

Figure 8. Meet the yaw angle when the value is square wave and $u_{d}=1 \mathrm{~m} / \mathrm{s}$

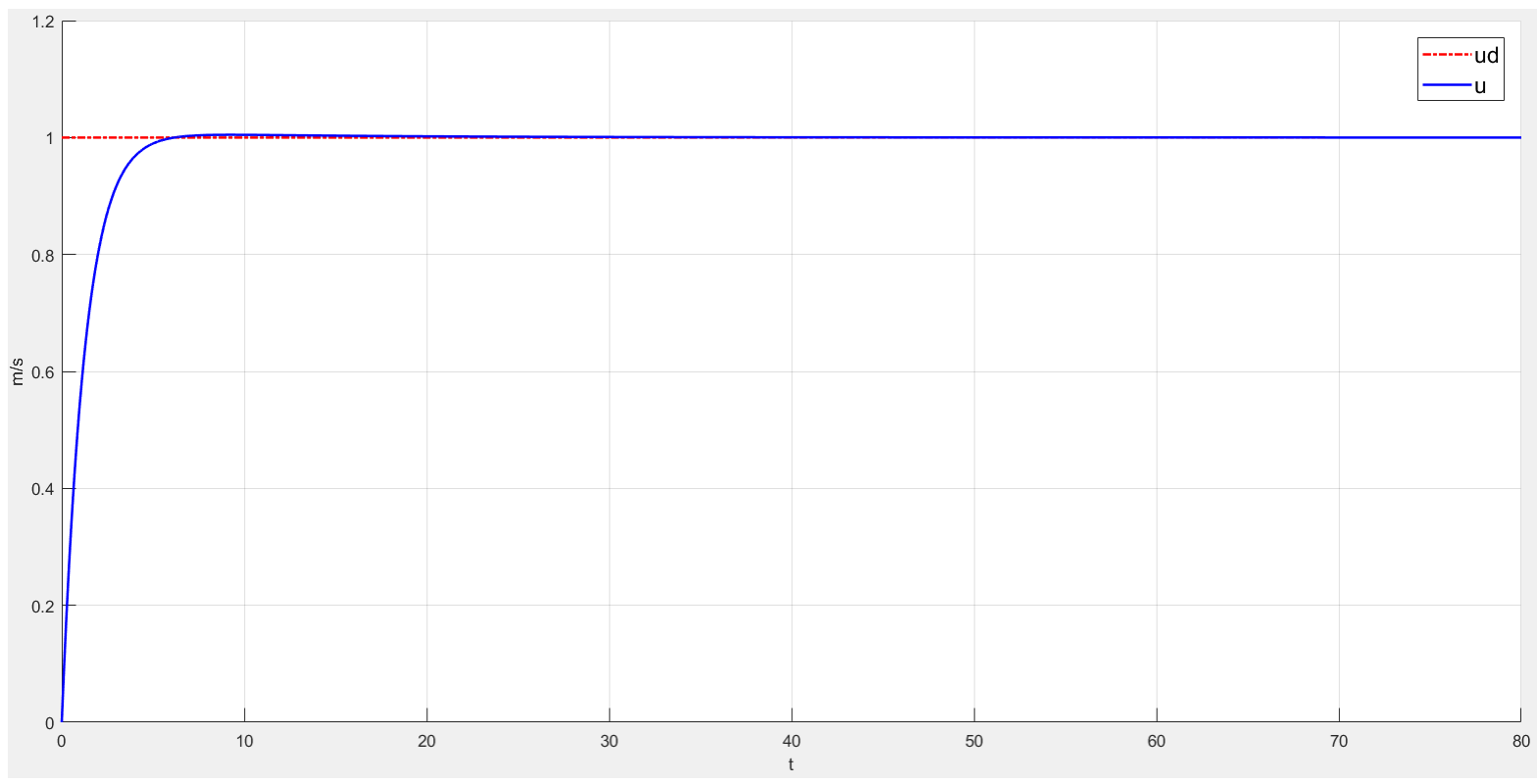

Figure 9. Simulate velocity when the yaw angle changes with square waves

Figure 9 describes meeting the velocity when the yaw angle changes with the square wave and the value of $u_{d}=1 \mathrm{~m} / \mathrm{s}$. This result shows that PI and PD controllers are not affected by each other when controlled simultaneously, have independence and have little effect on each other because we have removed the Coriolis 
component, however this is only true at a small velocity.

\section{CONCLUSIONS}

In summary, through the results of the design of the control algorithm using PID and simulation, we see that the combination of the guide and the controller has solved the core problems in the problem of self-driving for the ship. The results obtained are very positive and practically consistent.

In addition, the simulation has also shown the impact of the components in the Guidance set, as well as the optimization between control algorithms to orbital quality will help the designer understand the nature of the parameters to fine-tune them accordingly and achieve the desired target.

\section{REFERENCES}

[1] Fossen TI, Marine Control Systems Guidance, Navigation and Control of Ships, Rigs and Underwater Vehicles, Marine Cybernetics AS, 2002.

[2] Fossen TI, Handbook of Marine Craft Hydrodynamics and Motion Control, Wiley, New York, 2011.

[3] Hauser J, \& Hindman R. Aggressive flight maneuvers, Proceedings of the 36th IEEE conference on decision \& control. IEEE, San Diego, CA, USA, p. 4186-4191, 1997.

[4] Kevin Klemens, Development and Evaluation of a USV Based Mapping System for Remote Sensing of Eelgrass Extent in Southern California, 2017.

[5] S. Wang, H. Jin, L. Meng, Trajectory Tracking for Underactuated UUV Using Terminal Sliding Mode Control, Chinese Control and Decision Conference (CCDC), 2016.

[6] Tran Ngoc Huy, Study on Optimized Guidance and Robust Control for the Ship Maneuvering, International Conference on Advanced Engineering Theory and Applications, 2017.

[7] X. Chen, Z. Liu, H. Hu, L. Wang, J. Dong, Backstepping Adaptive Sliding Mode Control for the USV Course Tracking System, 9th International Conference on Advanced Infocomm Technology (ICAIT), 2017.

[8] Y. Fan, X. Sun, G. Wang; C. Guo, On Fuzzy Self-adaptive PID Control for USV Course, 34th Chinese Control Conference (CCC), 2015. 


\section{BIOGRAPHIES OF AUTHORS}
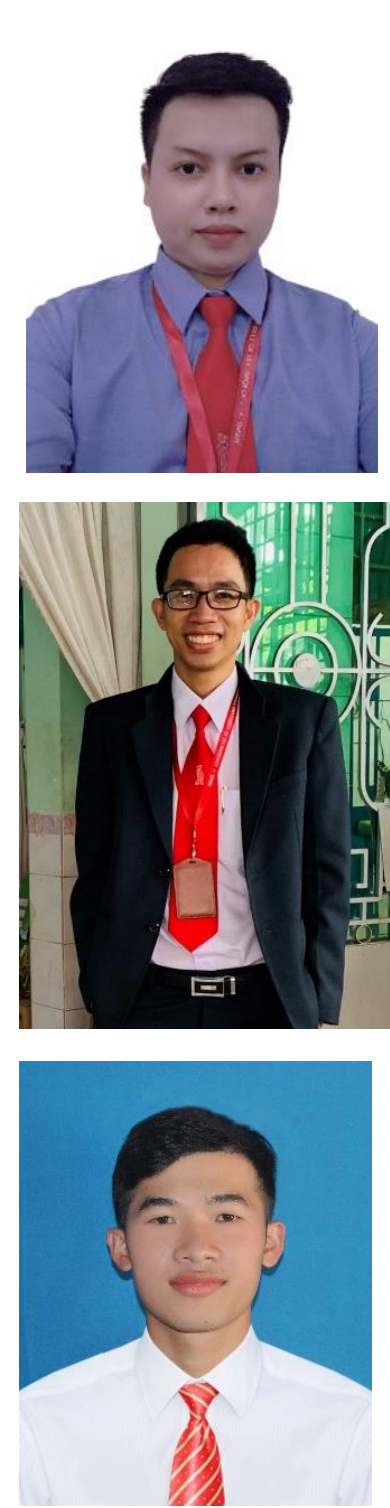

Full name: Nhat Duy Nguyen

Gender: Male

Date of birth: July 21, 1995

Nationality: Vietnamese.

Degree: Master

Specialization: mechanical engineering

Working unit: Dong Nai University of Technology.

Contact phone: 094.4567.298

Email: nguyennhatduy@dntu.edu.vn

1. Full name: Thanh - Lanh Le

2. Affiliation including postal code: Department of Technology, Dong Nai Technology University, Dong Nai, 760000, Viet Nam

3. email: lethanhlanh@dntu.edu.vn

4. Biography: Thanh-Lanh Le is a lecture and Vice Dean at the Department of Technology, Dong Nai Technology University, Bien Hoa City, Viet Nam. He received his M.S degrees in Electrical and Electronic engineering at Ho Chi Minh City University of Technology and Education, Ho Chi Minh, Viet Nam in 2015. He received his Ph.D in Electrical engineering at National Kaohsiung University of Science and Technology, Kaohsiung, Taiwan in 2020. He currently as an Editor of the International Journal of Research in Vocational Studies (IJRVOCAS). His research interests include Optimization System, the Intelligence of Things, Intelligent Power systems, and Optoelectronics, Photonics and Optical System.

Full name: Nam Thoi Le

Gender: Male

Date of birth: September 9, 1999

Nationality: Vietnamese.

Degree: Engineer

Specialization: Electrical engineering

Working unit: Dong Nai University of Technology.

Contact phone: 034.7564.705

Email: lenamthoi09@gmail.com 